\title{
Effects of Otitis Media on the Eustachian Tube: An Animal Experiment
}

\author{
Muzaffer Kiris ${ }^{1}$, Ergun Sevil ${ }^{2 *}$, Togay Muderris ${ }^{3}$ and Faruk Kiroglu ${ }^{4}$ \\ ${ }^{1}$ Department of Otolaryngology Head and Neck Surgery, Yildirim Beyazit University, Turkey \\ ${ }^{2}$ Department of Otolaryngology Head and Neck Surgery, Kanuni Training and Research Hospital, Turkey \\ ${ }^{3}$ Department of Otolaryngology Head and Neck Surgery, Bozyaka Training and Reserch Hospital, Turkey \\ ${ }^{4}$ Department of Otolaryngology Head and Neck Surgery, Van Yuzuncu Yil University, Turkey
}

Submission: December 21, 2019; Published: January 23, 2020

*Corresponding author: Ergun Sevil, Department of Otolaryngology Head and Neck Surgery, Kanuni Training and Research Hospital, Trabzon, Turkey

Abstract

Objective: To study the effect of the acute otitis media (AOM) on the middle ear mucosa and auditory tube in rabbits and to evaluate the results histologically.

Material Methods: The study was carried out on 35 adult rabbits (New Zealand white rabbits), weighing 1500-1800 grams. The middle ear was inoculated with a bacterial suspension containing S. aureus to induce acute otitis media. Based on the exposure time of 1, 2, 4, 12, 24 weeks, the animals were classified into five groups. The rabbits in the acute otitis media group were killed after they were exposed for 1 and 2 weeks (acute), four weeks (subacute), or 12 and 24 weeks (chronic), and they were compared with the control group. The left control middle ear, the right inoculated bulla and the eustachian tube were removed. Sections of the temporal bones were dissected on block by covering the middle ear cavity and nasopharynx passing through the eustachian tube.

Results: The goblet cells in the group AOM after one week was as dense as those in the control group. There was no difference between the groups in subepithelial edema, the number of eosinophils, subepithelial vasodilatation, intraepithelial gland formation and, subepithelial gland formation $(p>0.05)$. The gland volume and the goblet cells density were extended to peak two weeks after the inoculation since there was primarily a volume increase in the components of the mucous gland. There was a significant increase in the volume of the gland layer within at least 12 weeks after the inoculation of the bacterial middle ear, primarily since there was an increase in a volume of the components of the mucous gland. Although there was a decrease in the volume 12 weeks after acute infection, it was completely normalized after 24 weeks.

Conclusion: Acute otitis media directly affects the auditory tube. Besides, excessive mucus secretion and goblet cell proliferation included histologic changes of auditory tube mucosa

Keywords: Acute Otitis Media; Eustachian Tube; Goblet Cells

\section{Introduction}

One of the most common infectious diseases in all age groups is acute otitis media (AOM) characterized by symptoms of middle ear infection and proceeded manifestation of inflammation, and its pathogenesis is affected by host immune circumstance, structure and function of eustachian tube (ET), virulence and strain of pathogen, innate mucosal defense, and genetic vulnerability [1]. The most common four pathogens seen in AOM are Haemophilus influenzae, morexella catarrhalis, Streptococcus pneumoniae, and Staphylococcus aureus. Viruses, especially Rhinovirus and Adenoviruses, are identified as pathogens in up to $20 \%$ of patients [2]. Only if the balance is shifted inside, the eustachian will be used for these bacteria to colonize and invade the middle ear. After the change of the mucosal conditions in the host leading to the proliferation of the bacteria caused by the viral infection of an upper respiratory tract, this event will generally occur.

The eustachian tube is a small canal which extents along the tympanic cavity with the nasopharynx, helps normal hearing equalize the middle ear pressure (ventilation), drains the secretions from the middle ear to the nasopharynx (cleans), and avoids the aspiration to the middle ear (protection). Negative middle ear pressure is caused by ET dysfunction, yielding effusion. However, the exact mechanism that leads to rapid changes 
observed with AOM, including cytokine release, derangement of middle ear gas respiration, negative pressure, local immune response, and microbial colonization, has not been certainly understood $[3,4]$. This study aims to study the effect of the AOM on the middle ear mucosa and auditory tube in rabbits and to evaluate the results histologically.

\section{Materials and Methods}

The study was carried out on 35 adult rabbits (New Zealand white rabbits), weighing 1500-1800 grams. Before the experiment was conducted, the tympanic membrane was checked to exclude rabbits suffering from middle ear disease. All procedures were performed in conformity with the 1986 Strasbourg International Declaration of Animal Rights under veterinary supervision at the Medical Sciences Experimental Research and Practice Center following the ethical committee's approval.

Based on the exposure time of 1, 2, 4, 12, 24 weeks, the animals were classified into five groups. The rabbits in the acute otitis media group were killed after they were exposed for 1 and 2 weeks (acute), four weeks (subacute), or 12 and 24 weeks (chronic), and they were compared with the control group. Each tympanic membrane was studied using an operating microscope before death, excluding the obvious middle ear disease. The exclusion criteria included a purulent discharge in the external ear.

Anesthesia consisting of $5 \mathrm{mg} / \mathrm{kg}$ xylazine hydrochloride (Ksilazol; Provet Veteriner Urunleri Sanayi, Istanbul, Turkey) and $50 \mathrm{mg} / \mathrm{kg}$ ketamine (Ketalar; Eczacibasi Warner-Lambert, Istanbul, Turkey) was administered to all rabbits by intraperitoneally (i.p.) injection before the procedure. Subjects with no evident infection and perforation in the tympanic membrane at otomicroscopic examination were included in the study.

A fine needle was passed through the bony bulla wall to inoculate the middle ear with a bacterial suspension containing S. aureus ( $1 / 2$ Mc Farland (200 x $106 \mathrm{CFU} / \mathrm{ml})$ strain NCTC-8330 at $0.5 \mathrm{~mL}$ intratympanic ally to induce acute otitis media. The shaminoculated suspension medium was the left tympanic bulla of the rabbits, which acted as normal controls. AOM was expanded for 48 hours. Hyperemia of the tympanic membrane was observed after the second day the macroscope. The $S$. aureus growth was confirmed based on the culture. No antibiotics were applied to the rabbits in this course. On weeks $1,2,4,12$, and 24 , seven rabbits were randomly selected and killed on each occasion. Euthanasia was accomplished with an intraperitoneal injection of $100 \mathrm{mg}$ sodium pentobarbital, as an anesthetic agent, before the dissection of tympanic bone. Rabbits that were killed within 3 and 6 months were observed using otomicroscopy every 14 days to eliminate the spontaneous infection, and otomicroscopy was used to examine all rabbits on day 4 to ensure that there is established infection. The left control middle ear, the right inoculated bulla and the eustachian tube were removed. Sections of the temporal bones were dissected on block by covering the middle ear cavity and nasopharynx passing through the eustachian tube

\section{Histologic Examination}

Then, the cross-sections were taken by 5-10 micrometer intervals, parallel to the longitudinal axis of the eustachian tube. Each section was embedded in the paraffin block, and lams were prepared. They have been kept in a $4 \%$ formaldehyde solution for 48 hours and decalcified in 15\% formic acid. The taken slices are stained with hematoxylin and eosin, and examined for mucosal, submucosal, and peri tubal inflammations under the light microscope. All such structures were counted in every stained section with a magnification of $\mathrm{x} 200$ for determination of the density of tubal mucous and serous gland acini.

The numbers of eosinophils, lymphocytes and polymorphonuclear leukocytes (PMNLs), submucosal edema, submucosal vasodilatation, formation of mucosal gland and the formation of submucosal gland among the groups were assessed under a light microscope at a magnification of 200.

a. The goblet cell density was measured as low i.e., goblet cells $5 \%$, intermediate i.e., $6 \%-10 \%$, or high i.e., $10 \%$.

b. The numbers of PMNLs, lymphocytes, and eosinophils were measured as low i.e., $0-5$, intermediate i.e., $6-10$, or high i.e., 10.

c. Vasodilatation and submucosal edema were grouped into absent, mild, or manifest.

d. The formation of the submucosal, and the mucosal glands was measured as 1, 2, above.

\section{Statistical Analysis}

The statistical method used in this research was the MannWhitney test, with the critical significance level of $p<0.05$. The Kruskal-Wallis test (nonparametric analysis of variance) was used to calculate differences among the related parameters in groups.

\section{Results}

The goblet cells and pseudostratified mucociliary respiratorylike epithelium were included in the ET of the control group. The goblet cells in the group induced to AOM after one week was as dense as those in the control group. More than 5 to 10 lymphocytes and 10 PMNLs were found in the sections which were composed of the isthmus and ET nasopharyngeal orifice (Figure 1). There was no difference between the groups in subepithelial edema, the number of eosinophils, subepithelial vasodilatation, intraepithelial gland formation and, subepithelial gland formation ( $p>0.05)$. 


\section{Global Journal of Otolaryngology}

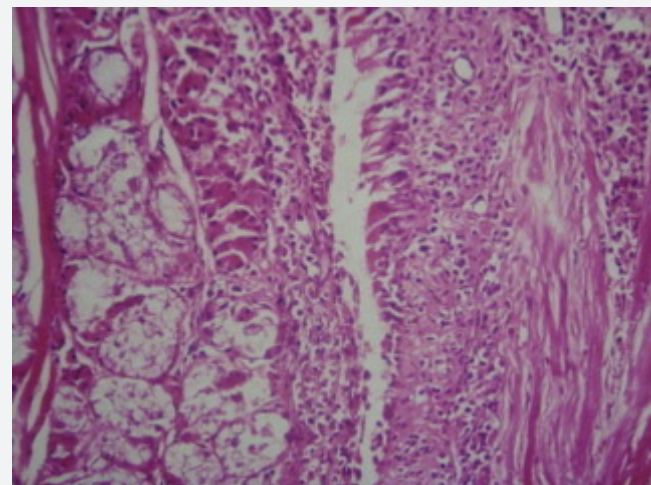

Figure 1: Light microscopy of peri tubal area. Showing infection findings in muscle layer.

The gland volume and the goblet cells density were extended to peak two weeks after the inoculation since there was primarily a volume increase in the components of the mucous gland. More than ten lymphocytes and 5 to 10 PMNLs were found in the sections which were composed of the isthmus, and ET nasopharyngeal orifice and lymphoid follicles were found (Figure 2). There were no significant differences between the subjects and the controls in the number of eosinophils, subepithelial vasodilatation, subepithelial edema, intraepithelial gland formation and subepithelial gland formation ( $p>0.05)$.

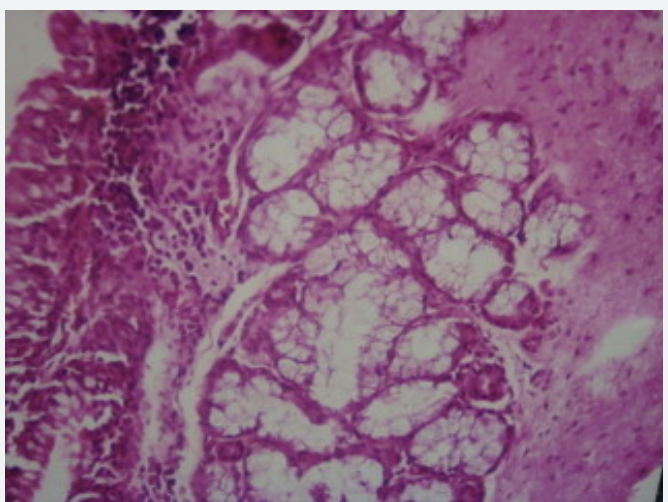

Figure 2: Showing cell infiltration of mucosa and submucosa.

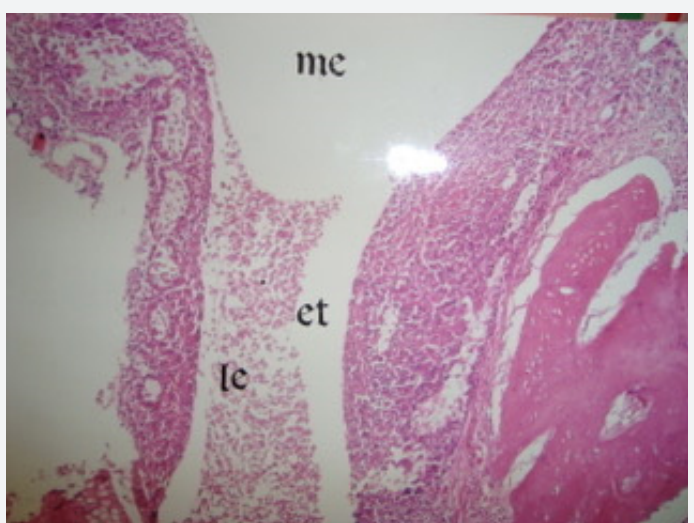

Figure 3: Profound polymorphonucleary leucocyte was formed in the sections of middle ear and the ET (me: middle ear, et: eustachian tube, le: polymorphonucleary leucocyte). 
There was a significant increase in the volume of the gland layer within at least 12 weeks after the inoculation of the bacterial middle ear, primarily since there was an increase in a volume of the components of the mucous gland (Figure 3). Although there was a decrease in the volume 12 weeks after acute infection, it was completely normalized after 24 weeks.

There was no change in the number and subtype of the gland acini, which indicates that hypertrophy of the gland cell, particularly the mucous gland cell caused to increase the volume. There was difference among the groups in the tympanic orifice $(\mathrm{P}=0.006)$, numbers of PMNLs at the nasopharyngeal orifice $(\mathrm{P}=0.029)$, middle segment of the ET $(\mathrm{P}=0.009)$, middle segment of the ET $(\mathrm{P}=0.015)$, density of goblet cells at the nasopharyngeal orifice $(\mathrm{P}=0.005)$, tympanic orifice $(\mathrm{P}=0.021)$, and the lymphocytes number at the nasopharyngeal orifice $(\mathrm{P}=0.017)$, while there were no differences in other parameters $(\mathrm{P}=0.056)$.

\section{Discussion}

The presence of an inflammatory substance in the middle ear, along with fever and other acute symptoms of the disease is called acute otitis media [5]. Acute otitis media typically follow an infection of the upper respiratory tract, which is often seen in children [6]. Despite medical therapy, the disease may continue to get worse, and it may end up with the secretory otitis media (SOM), chronic otitis media (COM), and recurrent otitis media (ROM) [7].

The immune immaturity of children below the age of 2 years and the enduring dysfunction of the ET cause a high recurrence rate [8]. The eustachian tube is highly effective in both the recovery and progress of acute otitis media. Long terming inflammation of ET is the most important reason for the AOM, particularly among the children below the age of 2 years. Impaired clearance, protection, and ventilation of the middle ear in this age group are caused by the small caliber of the ET as well as its horizontal direction. The poor enduring prognosis of AOM in children below the age of 2 years has been demonstrated in the recent prospective studies, reporting persistent otitis media with effusion (OME) and at least $50 \%$ of recurrences among $35 \%$ of children within six months after AOM.

In other words, if the protective function of ET is damaged during the early period of the disease, the formation of SOM and COM may develop due to the dysfunction of drainage. It is considered that the inflammation of peri tubal area affecting muscle during AOM may be responsible for the dysfunction of ET suppressing the contraction of the peri tubal muscle. The authors demonstrated that goblet cell depletion, loss of cilia, and squamous metaplasia of the ET mucosa were found after they were exposed to smoke in rats [9]. A study assessed the effects of reflux on the ET mucosa in rats. They claimed that the ET mucosa histopathology could be changed with nasopharyngeal exposure to experimental reflux [10]. The authors showed severe thickening of the mucosa of the ET orifice at the middle ear affected by SOM [11].

Several studies have shown an association between or increased staining of intracellular mucin or the increase of goblet cell density and goblet cell proliferation and differentiation and increase in mucus exocytosis, storage, and synthesis, which are regarded important for the evaluation of the potential tendency to extend the secretory otitis media [12]. Eustachian tube seems to play an effective role in the pathogenesis of OME since the middle ear is directly connected to the upper respiratory tract through the ET.

Lu et al. claimed that they had constituted the experimental inflammation by applying ethanol and acetic acid to the dog intestine, which shows that muscle contraction stimulated varies agents demonstrates less function than the normal muscles [13]. It is asserted that the inflammation may damage both myogenic and neural regulation of intestine muscle contractions. Furthermore, patients with ulcerative colitis or animal models created inflammation have demonstrated that the fascia contraction in the muscle decreases during inflammation period [14].

To figure out whether this effect depends on the inflammatory process that reduces muscle fibrils contraction functions or not, Wilcox et al. applied monocyte solutions to the hamster diaphragms and it is claimed that the inflammation decreases the muscle contraction by directly affecting the muscle fibrils [15]. The result of these studies is that inflammation involves the functional damage in both the smooth and skeletal muscles.

Herein, to detect the existence of inflammation of tensor veli palatini (TVP) muscle, located in the peri tubal area, AOM was experimentally induced in rabbits. According to our study, acute inflammatory cell infiltration of the soft tissue in peri tubal area due to the experimentally induced AOM could create movement restriction of the TVP that causes the dysfunction of the ET. During AOM, the inflammation of TVP muscle in peri tubal area may cause dysfunction of drainage of ET. Insufficient drainage function may delay the recovery of AOM as well as causing the ROM and SOM formation.

The middle ear is a part of respiratory mucosa, which has mucociliary system that has a crucial role in clearing the tympanic cavity [16]. The present paper showed that the ciliary activity of the middle ear mucosa in rabbits experimentally induced AOM was short, impaired and disrupted. The goblet cell density of eustachian tube that produces mucin, uphold mucosal integrity by producing antileukoprotease, and can lead to a more specific antimicrobial event through release and synthesis of bactericidal lysozyme is increased with acute otitis media [17]. However, the auditory tube may be obstructed with increased density of 
goblet cells, which pathologically and correspondingly enhanced secretion. Therefore, mucociliary transport function and ventilation of the middle ear may be damaged. Following this, the infection process may continue, or secretory otitis media may develop.

The proliferation of actual goblet cell as well as storage, synthesis, and exocytosis of mucin are affected by the increase of the goblet cell density and intracellular mucin [18]. When the potential inclination to develop the secretory otitis media is defined, it seems that these entities are needful subjects when defining [19]. Tos and Caye-Thomasen [12] found an increase in goblet cell density of the auditory tube within six months after acute otitis media. Kong et al. demonstrated that there was a slow increase in the number of goblet cell based on the exposure time to cigarette smoke in the middle ear and auditory tube [18]. Production of mucus immensely being due to population growth of goblet cell may worsen the function of an auditory tube, which can be observed after acute otitis media and thus predispose, continue, or complicate middle ear disease.

The limitation of this study is that the acute inflammation cascade was highly effective during the early stage, whereas it was important to increase the density of goblet cells in the late stage. A larger, long-term study is necessary for determining the role of this process. Then, the density of goblet cells and cilia function should be analyzed under electron microscopy.

\section{Conclusion}

In conclusion, acute otitis media directly affects the auditory tube. Besides, excessive mucus secretion and goblet cell proliferation included histologic changes of auditory tube mucosa. In the current study, the volume, number of gland acini, and composition were studied while the number of duct openings in the tube epithelium, which corresponds to the number of individual glands, was not considered. Further studies overall mounted eustachian tube mucosae are required to determine the number of glands ideally and more exactly.

\section{References}

1. Zhang S (2006) An improved nonparametric approach for detecting differentially expressed genes with replicated microarray data. Stat Appl Genet Mol Biol 5.

2. Scholz H, Noack R (1998) Multicenter, randomized, double-blind comparison of erythromycin estolate versus amoxicillin for the treatment of acute otitis media in children. AOM Study Group. Eur J Clin Microbiol Infect Dis 17:470-478.

3. Bhutta MF, Thornton RB, Kirkham LS, Kerschner JE, Cheeseman MT (2017) Understanding the aetiology and resolution of chronic otitis media from animal and human studies. Dis Model Mech 10(11): 12891300.

4. Coates AOH, Thornton R, Langlands J, et al. (2008) The role of chronic infection in children with otitis media with effusion: evidence for intracellular persistence of bacteria. Otolaryngol Head Neck Surg 138(6): 778-781.

5. Liu K, Chen L, Kaur R, Pichichero ME (2013) Transcriptome signature in young children with acute otitis media due to nontypeable Haemophilus influenza. Int Immunol 25(6): 353-361.

6. Bauchner H, Marchant CD, Bisbee A, Heeren T, Wang B et al. (2006) Effectiveness of Centers for Disease Control and Prevention recommendations for outcomes of acute otitis media. Pediatrics 117: 1009-1017.

7. Akyıldız N (1998) Kulak Hastalıkları ve Mikrocerrahisi (Ear Diseases and Microsurgery). Ankara: Bilimsel Tip Yayınevi 241-55

8. Corbeel L (2007) What is new in otitis media? Eur J Pediatr 166(6): 511-519.

9. Lee IW, Goh EK, Roh HJ, Lee CH, Chung BJ, et al. (2006) Histologic changes in the eustachian tube mucosa of rats after short-term exposure to cigarette smoke. Otol Neurotol 27(3): 433-440.

10. Yazici ZM, Sari M, Uneri C, MidiA, Tugtepe H (2008) Histologic changes in eusthacian tube mucosa of rats after exposure to gastric reflux. Laryngoscope 118(5): 849-853.

11. Christov F, Gluth MB (2018) Histopathology of the Mucosa of Eustachian Tube Orifice at the middle ear in chronic otitis media with effusion: Possible insight into tuboplasty failure. Ann Otol Rhinol Laryngol 127(11): 817-822.

12. Caye Thomasen P, Tos M (2003) Eustachian tube goblet cell density during and after acute otitis media caused by streptococcus pneumonia: a morphometric analysis. Otol Neurotol 24: 365-370.

13. Lu G, Qian X, Berezin I, Telford GL, Huizinga JD, et al. (1997) Inflammation modulates in vitro colonic myoelectric and contractile activity and interstitial cells of Cajal. Am J Physiol 273: 1233-1245.

14. Sethi AK, Sarna SK (1991) Colonic motor activity in acute colitis in conscious dogs. Gastroenterology 100: 954-963.

15. Wilcox P, Osborne S, Bressler B (1992) Monocyte inflammatory mediators impair in vitro hamster diaphragm contractility. Am Rev Respir Dis 146: 462-466.

16. Ohashi Y, Nakai Y, Kihara S, Maruoka K, İkeoka H, et al. (1985) The ciliary activity of the middle ear lining functional and morphological observation. Auris Nasus Larynx 12: 123-125.

17. Hanamure Y, Lim DJ (1986) Normal distribution of lysozyme and lactoferrin- secreting cells in chinchilla tubotympanum. Am J Otolaryngol 4: 410-425.

18. Kong SK, Chon KM, Goh EK, Lee W, Lee J, et al. (2009) Histologic changes in the auditory tube mucosa of rats after long-term exposure to cigarette smoke. American Journal of Otolarngology Head and Neck Medicine and Surgery (30): 376-382.

19. Jany B, Gallup M, Tsuda T, Basbaum C (1991) Mucin gene expression in rat airways after infection and irritation. Biochem Biophys Res Commun 181: 1-8. 
(C) Commons Attribution 4.0 License

(C) DY DOI: 10.19080/GJO.2020.21.556066
Your next submission with Juniper Publishers will reach you the below assets

- Quality Editorial service

- Swift Peer Review

- Reprints availability

- E-prints Service

- Manuscript Podcast for convenient understanding

- Global attainment for your research

- Manuscript accessibility in different formats ( Pdf, E-pub, Full Text, Audio)

- Unceasing customer service

Track the below URL for one-step submission https://juniperpublishers.com/online-submission.php 